\title{
Alimentação de peixes piscívoros antes e após a formação do reservatório de Salto Caxias, Paraná, Brasil
}

\author{
Gisele Caroline Novakowski ${ }^{1,2}$, Norma Segatti Hahn ${ }^{1,2,3,4}$ \& Rosemara Fugi ${ }^{1,3}$ \\ Biota Neotropica $v 7$ (n2) - http://www.biotaneotropica.org.br/v7n2/pt/abstract?article +bn04107022007 \\ Recebido em 07/02/07 \\ Versão reformulada recebida em 29/05/07 \\ Publicado em 15/07/07 \\ ${ }^{1}$ Universidade Estadual de Maringá - UEM, Av. Colombo 5790, CEP 87020-900, Maringá, PR, Brasil \\ e-mail: gcnovakowski@yahoo.com.br, \\ ${ }^{2}$ Programa de Pós-Graduação em Ecologia de Ambientes Aquáticos Continentais - PEA \\ ${ }^{3}$ Núcleo de Pesquisas em Limnologia, Ictiologia e Aqüicultura - Nupélia \\ e-mail: rosemarafugi@gmail.com \\ ${ }^{4}$ Autor para correspondência: Norma SegattiHahn,e-mail: hahnns@nupelia.uem.br
}

\begin{abstract}
Novakowski, G.C., Hahn, N.S. \& Fugi, R. Feeding of piscivorous fish before and after the filling of the Salto Caxias Reservoir, Paraná State, Brazil. Biota Neotrop. May/Aug 2007 vol. 7, no. 2. http://www. biotaneotropica.org.br/v7n2/pt/abstract?article+bn04107022007. ISSN 1676-0603.

This study investigated the feeding patterns of piscivorous fish before and after the filling of Salto Caxias Reservoir, Paraná State, Brazil. Specifically, it was determined if the diet of Hoplias malabaricus, Oligosarcus longirostris, Rhamdia branneri and Rhamdia voulezi altered according to prey availability. Fish were sampled monthly from March 1997 to February 1998 (pre-impoundment period) and from March 2000 to February 2001 (post-impoundment period) within the reservoir. Stomach contents of 549 individuals were analyzed and the diet was described as occurrence and volumetric percentage of each item combined in a feeding index. The piscivorous habit was, overall, more evident in $H$. malabaricus, $O$. longirostris and $R$. voulezi. However, for R. branneri mollusks and crabs were more important than fish. After the impoundment, most consumed prey was Astyanax sp. b and Cyphocharax modestus for three of the predators. These prey species were more abundant after the impoundment. Then, there are evidences that the availability, abundance and vulnerability of prey in the reservoir environment are the most important factors influencing predator's choice.
\end{abstract}

Keywords: diet, piscivory, trophic opportunism, neotropical reservoir, Brazil.

Resumo

Novakowski, G.C., Hahn, N.S. \& Fugi, R. Alimentação de peixes piscívoros antes e após a formação do reservatório de Salto Caxias, Paraná, Brasil. Biota Neotrop. May/Aug 2007 vol. 7, no. 2. http://www. biotaneotropica.org.br/v7n2/pt/abstract?article+bn04107022007. ISSN 1676-0603.

Este trabalho investigou padrões alimentares de peixes piscívoros antes e após a formação do reservatório de Salto Caxias, Paraná, Brasil, com base em estudos da dieta de Hoplias malabaricus, Oligosarcus longirostris, Rhamdia branneri e Rhamdia voulezi, e da disponibilidade de presas. As coletas foram realizadas mensalmente no período de março de 1997 a fevereiro de 1998 (pré-represamento) e de março de 2000 a fevereiro de 2001 (pós-represamento), no local do reservatório. Foram analisados 549 conteúdos estomacais e a composição da dieta foi descrita através das porcentagens de ocorrência e volume de cada item alimentar, as quais foram combinadas por um índice alimentar. O hábito piscívoro foi, em geral, mais evidente para $H$. malabaricus, $O$. longirostris e $R$. voulezi, enquanto que para $R$. branneri moluscos e crustáceos foram mais importantes que peixes. Após o represamento os peixes-presa mais consumidos, por três predadores, foram Astyanax sp. b e Cyphocharax modestus. Estas espécies estiveram entre as forrageiras mais capturadas após a formação do reservatório. Assim, conclui-se que mais do que uma preferência, o consumo por determinada presa é reflexo de sua disponibilidade, abundância e vulnerabilidade no ambiente represado.

Palavras-chave: dieta, piscivoria, oportunismo trófico, reservatório neotropical, Brasil. 


\section{Introdução}

Os peixes piscívoros, como predadores de topo em ecossistemas de água doce, exercem impacto direto ou indireto sobre a biota e qualidade da água (Nowlin et al. 2006) e segundo Carpenter \& Kitchell (1993), este efeito top-down tem sido denominado de cascata trófica. Neste contexto, os piscívoros constituem-se em objeto de inúmeros estudos, principalmente com o intuito de avaliar o efeito da predação sobre as populações de espécies-presa (Nilsson 1978). Além disso, essas espécies têm importância básica na manutenção da saúde de comunidades naturais por beneficiarem as populações através da remoção de indivíduos debilitados, menos ágeis e, portanto, mais vulneráveis (Simon 1983) sendo, por essa razão, considerados "melhoradores biológicos" (Popova 1978). No entanto, a presença de predadores pode perturbar o habitat da presa e a taxa de consumo alimentar, reduzindo conseqüentemente sua taxa de crescimento e sucesso reprodutivo (Wootton 1990). Peixes que apresentam essa estratégia interferem na composição qualitativa e quantitativa de presas (Persson et al. 1996), porém, são elementos necessários na ictiofauna, pois aumentam a estabilidade do ecossistema, regulando a sua própria abundância bem como a de diferentes espécies de presa (Popova 1978).

Em ambiente represado, principalmente nos primeiros anos de formação, um efeito inevitável é a alteração na abundância de espécies com a eventual eliminação de alguns componentes ictiofaunísticos. Algumas espécies fluviais desaparecem do reservatório, enquanto outras persistem e alteram suas densidades (Winston et al. 1991, Agostinho et al. 1992). Além disso, considerando que nos reservatórios a disponibilidade de matéria orgânica resultante da submersão e decomposição do material alóctone é alta, a produção em todos os níveis tróficos tende a aumentar, incluindo os peixes predadores (O'Brien 1990). Tem sido observado que peixes de pequeno porte, conhecidos como "espécies forrageiras" proliferam nos primeiros anos após o represamento de um rio, em especial as espécies eurífagas, como registrado no reservatório de Itaipu, PR, em reservatórios da bacia do rio Iguaçu, PR e no reservatório de Manso, MT (Hahn et al. 1998, Delariva 2002, Silva 2006). Nessas condições, é esperado que haja um incremento marcante de piscívoros (Agostinho et al. 1999), uma vez que seu alimento está amplamente disponível (Loureiro \& Hahn 1996). Wootton (1990) comenta que os peixes são bons amostradores do ambiente e enfatiza que seus conteúdos estomacais refletem a disponibilidade de alimento. Embora os peixes predadores utilizem um amplo espectro de presas, poucas predominam na dieta (Popova 1978), sendo o consumo elevado de determinadas presas associado principalmente às suas abundâncias no ambiente (Edds et al. 2002, Floeter \& Temming 2003). Embora vários fatores como tamanho e tipo da presa, local de refúgio e atividade dos predadores e presas influenciem a tomada do alimento, a disponibilidade tem sido considerada como fator primordial na dieta dos peixes (Kahilainen \& Lehtonen 2003). Considerando a teoria do forrageamento ótimo, Griffiths (1975) comenta que os peixes podem otimizar suas dietas de duas formas: capturando em maior proporção os organismos que constituem o recurso mais energético no ambiente (maximizadores de energia) ou atuando como a maioria dos peixes, através do consumo das presas mais abundantes (maximizadores de número). Esta seleção de alimento é segundo Kahilainen \& Lehtonen (2003), uma tática alimentar típica de piscívoros oportunistas.

Diante do acima exposto e considerando que esse trabalho foi desenvolvido antes e após a formação do reservatório de Salto Caxias, época em que as condições ambientais estavam totalmente alteradas e propícias a investigações de várias questões ecológicas, pretendeuse, através da análise da dieta de peixes potencialmente piscivoros, responder a seguinte pergunta: como estas espécies se comportam quanto aos padrões alimentares quando a disponibilidade de presas sofre flutuações?

\section{Materiais e Métodos}

\section{1. Área de estudo}

A bacia do rio Iguaçu, especialmente a área de influência do reservatório de Salto Caxias, localiza-se na região sudoeste do estado do Paraná $\left(25^{\circ} 32^{\prime} \mathrm{S}\right.$ e $53^{\circ} 30^{\prime} \mathrm{W}$; $25^{\circ} 35^{\prime} \mathrm{S}$ e $\left.53^{\circ} 06^{\prime} \mathrm{W}\right)$, na divisa entre os municípios de Capitão Leônidas Marques e Nova Prata do Iguaçu. O rio Iguaçu foi interceptado em novembro/98 para formação do reservatório de Salto Caxias, o quinto da série de reservatórios do médio rio Iguaçu, que ocupam aproximadamente $41 \%$ de seu curso total (Júlio Jr. et al. 1997). O reservatório está localizado à aproximadamente $180 \mathrm{~km}$ a montante das cataratas do Iguaçu e a $100 \mathrm{~km}$ a jusante da barragem do reservatório de Salto Osório. Possui uma área alagada de $124 \mathrm{~km}^{2}$. Os locais de amostragem antes (profundidade média $=7,7 \mathrm{~m}$ ) e após a formação do reservatório (profundidade média $=20 \mathrm{~m}$ ) são apresentados na Figura 1. Nesse estudo, os exemplares provenientes dos diferentes locais de coleta foram agrupados para análise dos dados.

\section{Amostragem e análise dos dados}

As coletas dos peixes foram realizadas mensalmente no período de março de 1997 a fevereiro de 1998 (fase de pré-represamento) e de março de 2000 a fevereiro de 2001 (fase de pós- represamento, que corresponde ao segundo ano de formação do reservatório). Os peixes foram amostrados com três baterias de 15 redes de espera, com malhagens variando de 3 a $16 \mathrm{~cm}$ entre nós opostos, operadas simultaneamente na superfície, no fundo e nas margens. Os equipamentos de pesca ficaram expostos, em cada estação de coleta, por 24 horas,

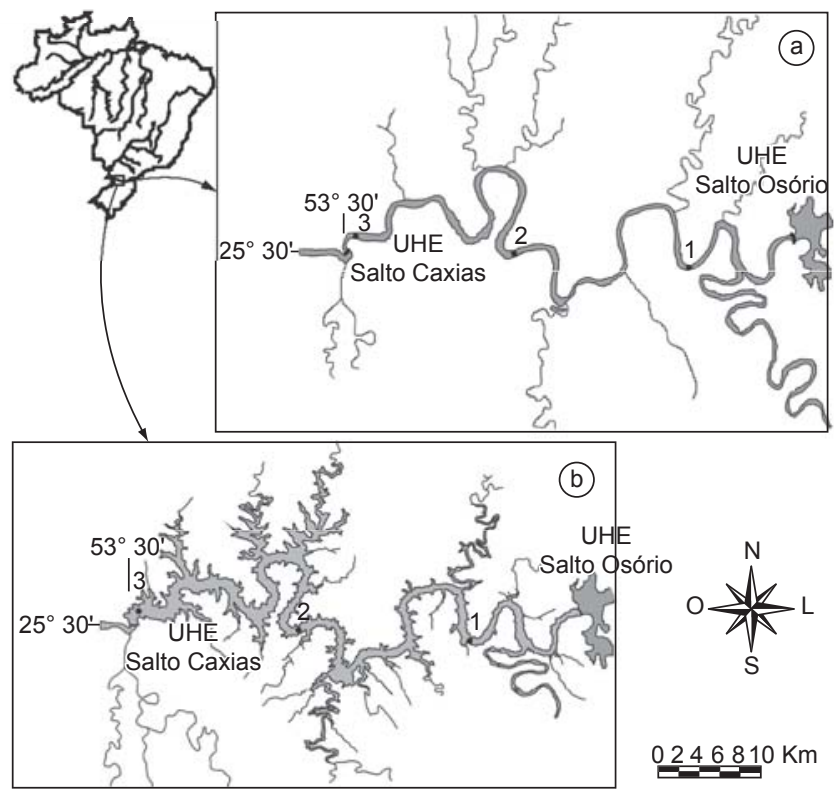

Figura 1. Mapa da área estudada. a) Rio Iguaçu; e b) reservatório de Salto Caxias, Paraná, Brasil. 1 = zona fluvial do reservatório; 2 = zona de transição; e 3 = zona lacustre.

Figure 1. Map of the studied area. a) Iguaçu River; and b) and Salto Caxias Reservoir, Paraná State, Brazil. 1 = riverine zone; 2 = transitional zone; and 3 = lacustrine zone. 
com revistas ao amanhecer ( 8 horas), ao entardecer (16 horas) e à noite (22 horas).

Após as despescas e obtenção de dados de comprimento padrão $(\mathrm{CP}=\mathrm{cm})$ e peso total $(\mathrm{Pt}=\mathrm{g})$, os peixes foram eviscerados e os estômagos retirados e preservados em formol $4 \%$. Para as análises foram considerados somente indivíduos adultos, a fim de evitar qualquer comprometimento dos dados em função de alterações ontogenéticas na dieta. As demais espécies capturadas na pesca experimental foram armazenadas em tambores com formol $4 \%$ e posteriormente utilizadas para avaliação da abundância da ictiofauna.

A dominância dos peixes no reservatório foi avaliada através da curva espécie-abundância ("Whittaker plots"), utilizando-se dados de captura por unidade de esforço (CPUE), através do seguinte cálculo:

$$
\text { CPUEn }=\mathrm{N} /\left(\mathrm{m}^{2} \times \mathrm{h}\right)
$$

onde: CPUEn = captura por unidade de esforço em número de indivíduos; $\mathrm{N}$ = número de indivíduos capturados por espécie; $\mathrm{m}^{2}$ = área das redes de espera; $\mathrm{h}=$ tempo de exposição das redes de espera.

As quatro espécies estudadas (Hoplias malabaricus, Oligosarcus longirostris, Rhamdia branneri e Rhamdia voulezi) possuem exemplares testemunhos depositados na coleção de peixes do Núcleo de Pesquisas em Limnologia, Ictiologia e Aquicultura (Nupélia/UEM) (Tabela 1).

Os conteúdos estomacais foram analisados e as presas foram identificadas até o nível taxonômico mais inferior possível, sendo as presas digeridas excluídas das análises. A importância das presas na dieta dos predadores foi estimada pelos métodos de freqüência de ocorrência e volumétrico (Hyslop 1980) e os dados foram sumarizados através do Índice Alimentar (IAi) (Kawakami \& Vazzoler 1980):

$$
\mathrm{IAi}=100(\% \mathrm{~F} . \% \mathrm{~V} / \Sigma \% \mathrm{~F} . \% \mathrm{~V})
$$

onde: $\mathrm{F}$ = freqüência de ocorrência de determinado item na dieta $(\%)$; $\mathrm{V}=$ volume de determinado item na dieta (\%). O volume das presas foi obtido através do deslocamento da coluna de água utilizando-se uma bateria de provetas graduadas.

\section{Resultados}

Com base na análise de 549 conteúdos estomacais das quatro espécies predadoras (169 da fase de pré-represamento e 380 da fase de pós-represamento), foi possível verificar que todas incluíram peixes em suas dietas, e com exceção de $H$. malabaricus, as demais espécies também consumiram invertebrados em pelo menos uma das fases consideradas (Tabela 2).

$\mathrm{Na}$ fase de pré-represamento $H$. malabaricus consumiu nove diferentes espécies de peixes-presa, sendo que Crenicichla spp. representou $69,4 \%$ dos valores do IAi. Após o represamento a contribuição de Crenicichla spp. decresceu para 2,9\% e Cyphocharax modestus passou a ser a presa mais consumida $(70,5 \%)$ (Tabela 2). Embora a dieta de $O$. longirostris tenha sido composta por nove diferentes tipos de peixes-presa antes do represamento, insetos (principalmente Coleoptera terrestre) foi o recurso alimentar mais importante, contribuindo com $69,1 \%$ da dieta. Dentre os peixes consumidos, apenas Astyanax sp. b teve uma participação relevante, representando 20,9\% dos valores do IAi. Após o represamento esta espécie passou a apresentar estratégia exclusivamente piscívora e duas espécies de peixes-presa dominaram a dieta. Cyphocharax modestus, não consumida na fase anterior, foi a mais predada $(52,0 \%)$, seguida por Astyanax sp. b (36,6\%). Destaca-se que sete espécies de peixes-presa consumidas na fase de pré-represamento não foram registradas nos conteúdos estomacais após o represamento, enquanto outras quatro foram consumidas apenas após este evento e os insetos praticamente desapareceram (Tabela 2).

A dieta de $R$. branneri foi composta basicamente por invertebrados, principalmente moluscos $(67,5 \%)$ e pelo caranguejo Aegla sp. $(26,1 \%)$ na fase de pré-represamento. Após esse evento, os moluscos não foram mais registrados nos conteúdos estomacais, enquanto que Aegla sp. passou a ser alimento quase que exclusivo, representando 93,6\% dos valores do IAi. O item peixes foi pouco explorado por esta espécie, independente da fase considerada (Tabela 2). Por outro lado, $R$. voulezi que apresentou uma dieta mista, composta principalmente por Aegla sp. (37,6\%) e Crenicichla spp. $(28,2 \%)$ na fase de pré-represamento, passou a explorar mais o item peixes após o represamento, ampliando seu espectro de três para seis novas espécies de peixes-presa, sendo Astyanax sp. b a mais consumida $(80,6 \%)$ (Tabela 2).

Considerando todo o período de estudos (pré e pós-represamento), foram registradas durante a pesca experimental, 52 espécies de peixes na área de influência do reservatório de Salto Caxias, sendo que as mais abundantes foram três espécies de lambaris, Astyanax sp. b, Astyanax sp. c e Astyanax altiparanae, seguidas pelo curimatídeo C. modestus (Figura 2). Destaca-se que a primeira e a última corresponderam às presas mais consumidas. Considerando a trajetória temporal na abundância dessas espécies, observa-se um incremento marcante nas capturas das quatro espécies, após a formação do reservatório, sendo o maior incremento verificado para Astyanax sp. c e C. modestus (Figura 3).

\section{Discussão}

As quatro espécies analisadas consumiram peixes em proporções diferentes umas das outras. Em função do predomínio de peixes nos conteúdos estomacais de $H$. malabaricus, de $O$. longirostris e de $R$. voulezi, em pelo menos uma das fases (rio e reservatório), estas espécies podem ser caracterizadas como piscívoras. Em contraste,

Tabela 1. Espécies estudadas, número de registro dos exemplares testemunho (Reg.), amplitude do comprimento padrão (CP, $\mathrm{cm}$ ) dos peixes analisados, número de estômagos analisados nas fases de pré (março/1997 a fevereiro/1998) e pós-represamento (março/2000 a fevereiro/2001) do reservatório de Salto Caxias, PR, Brasil. Nup = Núcleo de Pesquisas em Limnologia, Ictiologia e Aqüicultura.

Table 1. Studied species, register number of the voucher specimens (Reg.), range of standard length $(\mathrm{CP}, \mathrm{cm})$, N-pré (number of stomach analyzed in preimpoundment period - March 1997 to February 1998), N-pós (number of stomach analyzed in post-impoundment period March 2000 to February 2001) in the Salto Caxias Reservoir, Paraná State, Brazil. Nup = Center for Research in Limnology, Ichthyology and Aquaculture.

\begin{tabular}{lllrr}
\hline \multicolumn{1}{c}{ Espécies } & \multicolumn{1}{c}{ Reg. } & CP & N-pré & N-pós \\
\hline Hoplias malabaricus (Bloch 1794) & Nup 687 (3 ex.) & $18,2-50,5$ & 53 & 184 \\
Oligosarcus longirostris Menezes \& Géry 1983 & Nup 721 (2 ex.) & $10,4-28,0$ & 95 & 148 \\
Rhamdia branneri Haseman 1911 & Nup 2448 (28 ex.) & $17,9-40,0$ & 12 & 17 \\
Rhamdia voulezi Haseman 1911 & Nup 1659 (1 ex.) & $14,9-29,5$ & 9 & 31 \\
\hline
\end{tabular}


Tabela 2. Composição da dieta de Hoplias malabaricus, Oligosarcus longirostris, Rhamdia branneri e Rhamdia voulezi nas fases de pré e pós-represamento do reservatório de Salto Caxias, rio Iguaçu, PR. Pré = fase anterior ao represamento e Pós = fase após o represamento; IAi = Indice Alimentar; * valores <0,1.

Table 2. Diet composition of Hoplias malabaricus, Oligosarcus longirostris, Rhamdia branneri and Rhamdia voulezi in the pre $=$ before impoundment and post $=$ after impoundment of the Salto Caxias Reservoir, Iguaçu River, Paraná State. Pré $=$ before impoundment and Pós = after impoundment; IAi = Feeding Index; $*$ values $<0.1$

\begin{tabular}{|c|c|c|c|c|c|c|c|c|}
\hline \multirow{2}{*}{$\begin{array}{c}\text { Predadores } \\
\text { Itens }\end{array}$} & \multicolumn{2}{|c|}{ H. malabaricus } & \multicolumn{2}{|c|}{ O. longirostris } & \multicolumn{2}{|c|}{ R. branneri } & \multicolumn{2}{|c|}{ R. voulezi } \\
\hline & Pré & Pós & Pré & Pós & Pré & Pós & Pré & Pós \\
\hline & IAi & IAi & IAi & IAi & $\mathbf{I A i}$ & IAi & IAi & IAi \\
\hline \multicolumn{9}{|l|}{ PEIXES } \\
\hline Astyanax altiparanae & 8,4 & 0,6 & 0,2 & 1,6 & - & 3,6 & - & 1,0 \\
\hline Apareiodon vitattus & 2,3 & $*$ & - & 1,6 & - & - & - & 0,2 \\
\hline Astyanax sp. b & - & 17,7 & 20,9 & 36,6 & 0,2 & 2,5 & - & 80,6 \\
\hline Astyanax sp. c & 1,0 & 6,3 & - & 6,6 & - & - & - & 4,9 \\
\hline Cyphocharax modestus & 0,6 & 70,5 & - & 52,0 & - & - & - & 1,1 \\
\hline Characidae & - & - & - & - & - & - & 15,7 & - \\
\hline Corydoras paleatus & - & $*$ & - & - & - & - & - & 0,4 \\
\hline Crenicichla spp. & 69,4 & 2,9 & 1,6 & - & - & - & 28,2 & - \\
\hline Geophagus brasiliensis & - & 0,1 & - & - & - & - & - & - \\
\hline Glanidium ribeiroi & - & - & 0,5 & - & - & - & - & - \\
\hline Gymnotus carapo & 5,7 & 1,4 & 0,3 & - & - & - & - & - \\
\hline Oligosarcus longirostris & - & - & - & 1,6 & - & - & - & - \\
\hline Pimelodus spp. & 10,9 & 0,4 & 5,1 & - & 1,2 & - & - & - \\
\hline Psalidodon gymnodontus & 1,5 & - & - & - & - & - & - & - \\
\hline Rhamdia spp. & - & - & 0,3 & - & - & - & - & - \\
\hline Tatia sp. & 0,1 & - & 0,1 & - & - & - & - & - \\
\hline Trichomycterus spp. & - & - & 0,9 & - & - & - & - & - \\
\hline Hypostomus spp. & - & - & - & - & - & - & 7,8 & - \\
\hline \multicolumn{9}{|l|}{ INVERTEBRADOS } \\
\hline Insetos & - & - & 69,1 & 0,1 & - & 0,1 & - & 0,1 \\
\hline Aegla sp. & - & - & - & - & 26,1 & 93,6 & 37,6 & 0,1 \\
\hline Mollusca & - & - & - & - & 67,5 & - & 3,1 & - \\
\hline VEGETAIS & - & - & 1,1 & - & 3,1 & 0,2 & 1,9 & - \\
\hline Detrito & - & - & - & - & 1,9 & - & - & - \\
\hline
\end{tabular}

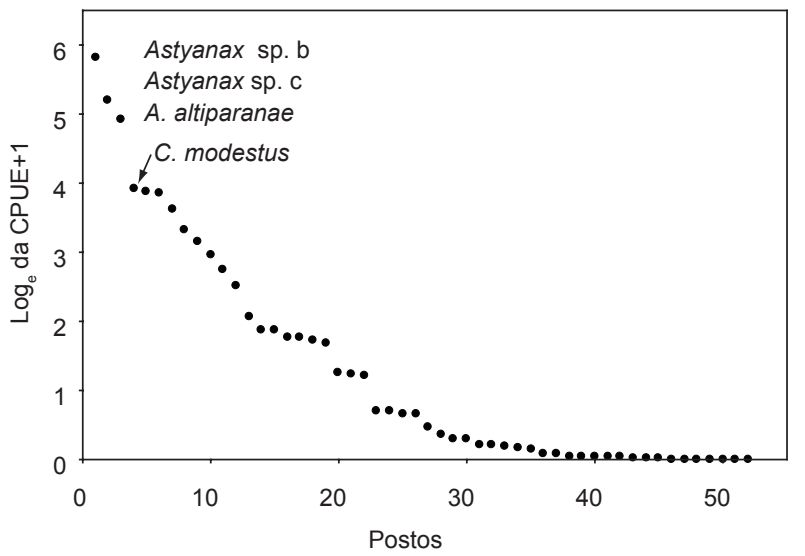

Figura 2. Curva espécie abundância da comunidade íctica do reservatório de Salto Caxias, rio Iguaçu, PR, para todo o período de amostragem.

Figure 2. Rank-abundance curve for fish assemblage of the Salto Caxias Reservoir, Iguaçu River, PR, during the entire studied period.

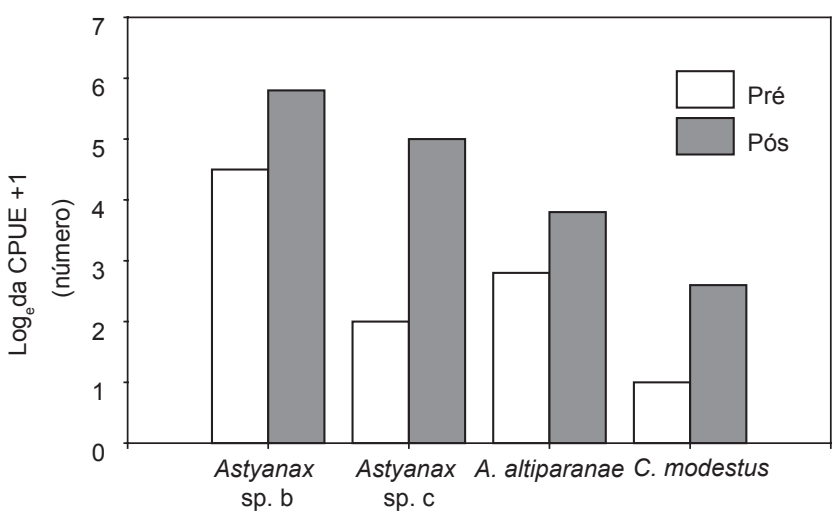

Figura 3. Variação temporal da captura por unidade de esforço (CPUE) das quatro espécies de peixes mais abundantes no reservatório de Salto Caxias, rio Iguaçu, PR; pré = fase anterior ao represamento e pós $=$ fase após o represamento.

Figure 3. Temporal variation in the caught (CPUE) of the four most abundant fish species in the Salto Caxias Reservoir, Iguaçu River, PR; pre = before impoundment and post $=$ after impoundment . 
$R$. branneri mostrou preferência por macroinvertebrados, embora algumas espécies de peixes tenham ocorrido esporadicamente nos estômagos.

O hábito essencialmente piscívoro de $H$. malabaricus tem sido registrado em vários estudos (Faccio \& Torres 1988, Canan et al. 1997). No reservatório de Segredo, Loureiro \& Hahn (1996) relatam que desde os estágios jovens a espécie já apresenta estratégia totalmente piscívora. Em contraste, Gurgel (2005) estudando comunidades de peixes de um trecho do rio Ceará Mirim, RN, verificou que $H$. malabaricus consumiu maior proporção de crustáceos, representando $58 \%$ de sua dieta. Para O. longirostris do reservatório de Segredo, bacia do rio Iguaçu, PR (Gealh \& Hahn 1998) e para Oligosarcus paranensis do reservatório de Capivari, PR (Abelha et al. 2005) o hábito essencialmente piscívoro também é mencionado. Nota-se, entretanto, que na fase de pré-represamento $O$. longirostris consumiu além de peixes uma quantidade considerável de insetos. Araújo et al. (2005) registraram também, elevado consumo de insetos por Oligosarcus hepsetus no reservatório de Lajes, RJ, enquanto Hartz et al. (1996) mencionam que Oligosarcus jenynsii, além de peixes, consumiu grande quantidade de camarões na lagoa Caconde, RS. Para $R$. branneri e $R$. voulezi poucos estudos referentes à alimentação foram realizados, sendo as informações restritas ao reservatório de Segredo, rio Iguaçu, PR, onde foi registrado hábito piscívoro para ambas, que complementaram suas dietas com aproximadamente 25\% de crustáceos (Hahn et al. 1997). Para a congênere Rhamdia quelen o hábito piscívoro foi registrado no reservatório de Corumbá, GO (Luz-Agostinho et al. 2006) e no reservatório de Capivari, PR (Abelha et al. 2005).

As variações temporais encontradas na dieta de $H$. malabaricus, $O$. longirostris e $R$. voulezi considerando-se as fases de pré e pósrepresamento, estão estreitamente associadas ao incremento na abundância de espécies forrageiras, principalmente de Astyanax sp. b e $C$. modestus. O sucesso destas espécies de pequeno porte, particularmente as do gênero Astyanax, nos reservatórios da bacia do rio Paraná, tem sido atribuído à estratégia reprodutiva, como a produção de pequenos ovos, elevada fecundidade, embriogênese curta e curto tempo de eclosão das larvas, além de uma elevada flexibilidade alimentar (Agostinho et al. 1999).

A relação entre a dieta dos piscívoros do reservatório de Salto Caxias e a abundância e disponibilidade de peixes-presa pode ser evidenciada pela alteração marcante na dieta de H. malabaricus, $O$. longirostris e R. voulezi. Embora espécies do gênero Astyanax já fossem abundantes antes do represamento, o incremento de Astyanax sp. b e de $C$. modestus parece ter contribuído para que a dieta destes predadores tenha se tornado restrita e especializada. Apesar de Astyanax sp. c e A. altiparanae terem sido mais abundantes que C. modestus, elas não se constituíram nas presas mais consumidas. É provável que aspectos morfológicos que comprometam a eficiência natatória, tais como, corpo relativamente mais baixo e mais robusto do que as espécies de Astyanax, respondam pela maior vulnerabilidade de $C$. modestus à predação.

Além disso, esse curimatídeo possui hábito detritívoro, vivendo mais associado ao substrato, e com o aumento da profundidade após o represamento, a população pode ter evadido e se concentrado em áreas mais rasas. Portanto, a predação mais acentuada sobre Astyanax sp. b certamente se deve à sua abundância, mas sobre $C$. modestus o fator mais importante deve estar associado à sua vulnerabilidade temporária. Entretanto, essas inferências carecem de estudos pormenorizados, como por exemplo, uma amostra maior de estômagos analisados e principalmente estudos experimentais.

Alterações na dieta de piscívoros em função do incremento de espécies forrageiras após represamentos têm sido registradas em vários reservatórios (Hahn et al. 1998, Gealh \& Hahn 1998). Um exemplo notável foi registrado no reservatório de Manso (MT), onde a dieta de Acestrorhynchus pantaneiro sofreu marcante alteração em função da explosão da forrageira Moenkhausia dichroura, que representava apenas $15 \%$ da dieta logo no início da formação do reservatório e passou a compor $95 \%$ no quarto ano após o represamento (Silva 2006).

Para $R$. branneri a alteração da dieta entre as fases de pré e pósrepresamento foi também expressiva. Embora essa espécie tenha apresentado estratégia diferente das demais, o desaparecimento de moluscos (bivalves e gastrópodos) nos conteúdos estomacais, seu alimento principal na fase rio, sugerem alterações marcantes na abundância desses organismos após a formação do reservatório. Enquanto muitos peixes mostram incremento nas capturas, como já discutido, os invertebrados bentônicos são prejudicados pela escassez de oxigênio a que são submetidos devido ao aumento da profundidade. Em se tratando de moluscos, que possuem pouca mobilidade, a transferência das populações para áreas mais propícias é quase impossível, ao contrário dos caranguejos Aegla sp., os quais se instalam em regiões mais rasas, como na foz dos tributários. De acordo com Baxter (1977), quando um rio é barrado, os organismos bentônicos característicos de ambientes lóticos tendem a desaparecer e eventualmente serem substituídos por aqueles com características lênticas. Ainda, segundo Takeda et al. (2005), a maior parte dos bivalves nativos é adaptada aos ambientes lóticos e quando confinados em reservatórios eles podem sobreviver durante um tempo, porém sofrem uma série de limitações que irão dificultar ou até interromper seu poder de reprodução e dispersão. No reservatório de Segredo, rio Iguaçu, PR, duas espécies de peixes malacófagas praticamente desapareceram do reservatório nos dois primeiros anos após o represamento, sendo este fato atribuído ao decréscimo de moluscos na área represada (Fugi 1988).

A piscivoria entre peixes é obrigatória para algumas espécies e oportunista para outras que atuam temporalmente, tomando vantagem da densidade elevada de uma determinada presa. Sem dúvida, a morfologia do aparato trófico afeta a performance forrageira do predador, porém, de acordo com Gerking (1994) quando surge uma fonte alternativa de alimento proveitosa, muitas espécies conseguem se beneficiar da oportunidade, de acordo com a teoria do forrageamento ótimo. Neste contexto, pode-se inferir que em ambiente recém-represado, mais do que uma preferência, o consumo de determinados tipos de alimento pelos predadores é reflexo da abundância, disponibilidade e vulnerabilidade das presas.

\section{Agradecimentos}

Os autores agradecem ao Nupélia (Núcleo de Pesquisas em Limnologia, Ictiologia e Aqüicultura) pela infra-estrutura concedida e ao convênio UEM/Nupélia/Copel pelo apoio financeiro.

\section{Referências Bibliográficas}

ABELHA, M.C.F., GOULART, E. \& PERETTI, D. 2005. Estrutura trófica e variação sazonal do espectro alimentar da assembléia de peixes do reservatório de Capivari, Paraná, Brasil. In Biocenoses em reservatórios: padrões espaciais e temporais (L. Rodrigues, S.M. Thomaz, A.A. Agostinho \& L.C. Gomes, eds.). RIMA, São Carlos, p.197-209.

AGOSTINHO, A.A., JÚLIO JR., H.F. \& BORGHETTI, J.R. 1992. Considerações sobre os impactos dos represamentos na ictiofauna e medidas para sua atenuação. Um estudo de caso: Reservatório de Itaipu. Unimar. 14:89-107.

AGOSTINHO, A.A., MIRANDA, L.E., BINI, L.M., GOMES, L.C., THOMAZ, S.M. \& SUZUKI, H. I. 1999. Patterns of colonization in neotropical reservoirs, and prognoses on aging. In Theoretical reservoir ecology and its applications (J. G. Tundisi \& M. Straskraba, eds.). International Institute of Ecology, São Carlos, p.227-265. 
ARAÚJO, F.G., ANDRADE, C.C., SANTOS, R.N., SANTOS, A.R.G.N. \& SANTOS, L.N. 2005. Spatial and seasonal changes in the diet of Oligosarcus hepsetus (Characiformes, Characidae) in a Brazilian Reservoir. Braz. J. Biol. 65(1):1-8.

BAXTER, R.M. 1977. Environmental effects of dams and impoundments. Annu. Rev. Ecol. Syst. 8:255-283.

CANAN, B., GURGEL, H.C.B., NASCIMENTO, R.S.S., BORGES, S.A.G. \& BARBIERI, G. 1997. Avaliação da comunidade de sete espécies de peixes da lagoa Boa Cicca, Nísia Floresta - RN. Rev. Ceres 64(256):604-616.

CARPENTER, S.R. \& KITCHELL, J.F. 1993. The trophic cascade in lakes. Cambridge University Press, Cambridge.

DELARIVA, R.L. 2002. Ecologia trófica da ictiofauna do rio Iguaçu-PR sob efeito do represamento de Salto Caxias. Tese de doutorado, Universidade Estadual de Maringá, Maringá.

EDDS, O.R., MATTHEWS, W.J. \& GELWICK, F.P. 2002. Resource use by large catfishes in a reservoir: is there evidence for interactive segregation and innate differences? J. Fish. Biol. 60(3):739-750.

FACCIO, I. \& TORRES, G.E. 1988. Regime alimentar de Hoplias malabaricus (Bloch, 1974) e H. lacerdae (Ribeiro, 1907) (Pisces, Erythrinidae) do reservatório de Três Marias, rio São Francisco, MG. In Associação Mineira de Aqüicultura. Coletânea de resumos dos encontros da Associação Mineira de Aqüicultura (AMA): 1982-1987. Brasília, DF: CODEVASF, p.64-65.

FLOETER, J. \& TEMMING, A. 2003. Explaining diet composition of North Sea cod (Gadus morhua L.): Prey size preference vs prey availability. Can. J. Fish. Aquat. Sci. 60:140-150.

FUGI, R. 1998. Ecologia alimentar de espécies endêmicas de lambaris do trecho médio da bacia do rio Iguaçu. Tese de Doutorado, Universidade Federal de São Carlos, SP.

GEALH, A.M. \& HAHN, N.S. 1998. Alimentação de Oligosarcus longirostris Menezes e Gèry (Osteichthyes, Acestrorhynchinae) do reservatório de Salto Segredo, Paraná, Brasil. Rev. Bras. Zool. 15(4):985-993.

GERKING, S.D. 1994. Feeding ecology of fishes. Academic Press, San Diego, California.

GRIFFITHS, D. 1975. Prey availability and food of predators. Ecology 56:1209-1214.

GURGEL, H.C.B. 2005. Alimentação da comunidade de peixes de um trecho do rio Ceará Mirim, em Umari, Taipu, Estado do Rio Grande do Norte, Brasil. Acta Sci. Anim. Sci. 27(2):229-233.

HAHN, N.S., FUGI, R., ALMEIDA, V.L.L., RUSSO, M.R. \& LOUREIRO, V.E. 1997. Dieta e atividade alimentar de peixes do reservatório de Segredo. In Reservatório de Segredo: bases ecológicas para o manejo (A.A. Agostinho \& L.C. Gomes, eds.) EDUEM, Maringá, p.141-162.

HAHN, N.S., AGOSTINHO, A.A., GOMES, L.C. \& BINI, L.M. 1998. Estrutura trófica da ictiofauna do reservatório de Itaipu (Paraná - Brasil) nos primeiros anos de sua formação. Interciência 23(5):229-235.

HARTZ, S.M., MARTINS, A. \& BARBIERI, G. 1996. Dinâmica da alimentação e dieta de Oligosarcus jenynsii (Gunther, 1864) na lagoa Caconde, Rio Grande do Sul, Brasil (Teleostei, Characidae). B. Inst. Pesca 23:21-29.
HYSLOP, E.M.S. 1980. Stomach contents analysis - a review of methods and their application. J. Fish. Biol. 17:411-429.

JÚLIO JR., H.F., BONECKER, C.C. \& AGOSTINHO, A.A. 1997. Reservatório de Segredo e sua inserção na bacia do rio Iguaçu. In Reservatório de Segredo: bases ecológicas para o manejo (A.A. Agostinho \& L.C. Gomes, eds.) EDUEM, Maringá, p.01-17.

KAHILAINEN, K. \& LEHTONEN, H. 2003. Piscivory and prey selection of four predator species in a whitefish dominated subarctic lake. J. Fish. Biol. 63:59-672

KAWAKAMI, E. \& G. VAZZOLER. 1980. Método gráfico e estimativa de índice alimentar aplicado no estudo da alimentação de peixes. Bol. Inst. Oceanogr. 29:205-207.

LOUREIRO, V.E. \& HAHN, N.S. 1996. Dieta e atividade alimentar da traíra, Hoplias malabaricus (Bloch, 1794) (Osteichthyes, Erythrinidae), nos primeiros anos de formação do reservatório de Segredo-PR. Acta Limnol. Brasil. 8:195-205.

LUZ-AGOSTINHO, K.D.G., BINI, L.M., FUGI, R., AGOSTINHO, A.A. \& JÚLIO Jr., H.F. 2006. Food spectrum and trophic structure of the ichthyofauna of Corumbá reservoir, Paraná river Basin, Brazil. Neotrop. Ichthyol. 4(1):61-68.

NILSSON, N.A. 1978. The role of size-biased predation in competition and interactive segregation in fish. In Ecology of freshwater fish production (S.D. Gerking, ed.). Blackwel Scientific, Oxford, p.303-325.

NOWLIN, W.H., DRENNER, R.W., GUCKENBERGER, K.R., LAUDEN, M.A., ALONSO, G.T., JOSEPH, E.F. \& SMITH, J.L. 2006. Gape limitation, prey size refuges and top-down impacts of piscivorous largemouth bass in shallow pond ecosystem. Hydrobiol. 563:357-369.

O'BRIEN, W.J. 1990. Perspectives on fish in reservoir limnology. In Reservoir Limnology: Ecology Perspectives (K.W. Thornton, B.L. Kimmel \& F.E. Payne, eds.). John Wiley, New York, p.209-225.

PERSSON, L., ANDERSON, J., WAHLSTRON, E. \& EKLOV, P. 1996. Size-specific interactions in lake ecosystems: predator gape-limitation and prey growth rate and mortality. Ecology 77:900-911.

POPOVA, O.A. 1978. The role of predaceous fish in ecosystems. In Ecology of freshwater fish production (S.D. Gerking, ed.). Blackwell Scientific, Oxford, p.215-249.

SILVA, G.C. 2006. Comportamento de forrageamento de Acestrorhynchus pantanteiro Menezes, 1992 no reservatório de Manso/MT. Dissertação de mestrado, Universidade Estadual de Maringá, Maringá.

SIMON, N. 1983. Predators and prey. J.M. Dent e Sons, London.

TAKEDA, A.M., MANSUR, M.C.D. \& FUJITA, D.S. 2005. Ocorrência de moluscos bivalves em diferentes reservatórios. In Biocenoses em reservatórios: padrões espaciais e temporais (L. Rodrigues, S.M. Thomaz, A.A. Agostinho \& L.C. Gomes, eds.). RIMA, São Carlos, p.161-167.

WINSTON, M.R., TAYLOR, C.M. \& PIGG, J. 1991. Upstream extirpation of four minnow species due to damming of a prairie stream. Trans. Am. Fish. Soc. 120:98-105.

WOOTTON, R.J. 1990. Ecology of teleost fishes. Chapman and Hall, London. 